Observation of a tricritical wedge filling transition in the 3D Ising model

This content has been downloaded from IOPscience. Please scroll down to see the full text. 2014 EPL 10826003

(http://iopscience.iop.org/0295-5075/108/2/26003)

View the table of contents for this issue, or go to the journal homepage for more

Download details:

IP Address: 150.214.182.116

This content was downloaded on 29/03/2016 at $14: 44$

Please note that terms and conditions apply. 


\title{
Observation of a tricritical wedge filling transition in the 3D Ising model
}

\author{
A. Rodríguez-Rivas ${ }^{1}$, J. M. Romero-Enrique ${ }^{1}$, L. F. Rull ${ }^{1}$ and A. Milchev ${ }^{2,3}$ \\ 1 Departamento de Física Atómica, Molecular y Nuclear, Área de Física Teórica, Universidad de Sevilla \\ Apartado de Correos 1065, 41080 Sevilla, Spain \\ 2 Institute for Physical Chemistry, Bulgarian Academy of Sciences - 1113 Sofia, Bulgaria \\ 3 Institut für Physik, Johannes Gutenberg-Universität Mainz - Staudinger Weg 7, D-55099 Mainz, Germany
}

received 28 July 2014; accepted in final form 6 October 2014

published online 27 October 2014

PACS 68.08.Bc - Wetting

PACS 68.35.Rh - Phase transitions and critical phenomena

PACS $64.60 . \mathrm{Kw}$ - Multicritical points

\begin{abstract}
In this letter we present evidences of the occurrence of a tricritical filling transition for an Ising model in a linear wedge. We perform Monte Carlo simulations in a double wedge where antisymmetric fields act at the top and bottom wedges, decorated with specific field acting only along the wegde axes. A finite-size scaling analysis of these simulations shows a novel critical phenomenon, which is distinct from the critical filling. We adapt to tricritical filling the phenomenological theory which successfully was applied to the finite-size analysis of the critical filling in this geometry, observing good agreement between the simulations and the theoretical predictions for tricritical filling.
\end{abstract}

Copyright (C) EPLA, 2014

Fluids adsorbed on micropatterned and sculpted solid substrates are known to exhibit phase transitions which differ from those observed at planar, homogeneous walls $[1-3]$. The simple 3D wedge geometry, characterized by a tilt angle $\alpha$, has been extensively studied in the past [3-26]. Thermodynamic arguments [27-29] show that the wedge in the presence of a saturated gas is completely filled with liquid provided that the contact angle $\theta$ is less than the tilt angle $\alpha$. This transition may be first-order or continuous (critical filling). For the latter, the characteristic lengthscales as the averaged interfacial height $\ell_{W}$, the correlation length $\xi_{y}$ along the wedge axis and the interfacial roughness $\xi_{\perp}$ diverge continuously as $\theta \rightarrow \alpha$ according to the power laws $[5,6]$

$\ell_{W} \sim(\theta-\alpha)^{-\beta_{W}}, \quad \xi_{y} \sim(\theta-\alpha)^{-\nu_{y}}, \quad \xi_{\perp} \sim(\theta-\alpha)^{-\zeta_{W} \nu_{y}}$,

where the critical exponents for short-ranged forces take the values $\beta_{W}=1 / 4, \nu_{y}=3 / 4$ and $\zeta_{W}=1 / 3$. In a shallow wedge, corresponding to small $\alpha$, the conditions for observing continuous wedge filling transition are less restrictive than for critical wetting at planar walls, and critical filling may occur even if the walls of the wedge show first-order wetting $[5,6]$. In more acute wedges the situation is more complicated. For example recently it has been proposed that if the wedge is acute enough, the filling transition may become of first order even if the wetting at the planar walls is continuous [16,17]. Furthermore, a phenomenological theory which takes into account the breather-mode interfacial fluctuations shows that the filling transition may change to be of first order by modifying the fluid-solid interactions close to the wedge bottom [13-15,24]. Simple heuristic arguments may be used to rationalize the latter results. At bulk coexistence and near the wedge filling transition, the gasliquid interface is locally flat and its position is determined by the midpoint interfacial height above the wedge bottom $\ell(y), y$ being the coordinate along the wedge axis $[5,6]$. If $\ell(y)$ changes by an amount $\Delta \ell(y)$, the macroscopic contribution to the excess surface free energy associated with a lateral section (i.e. fixed $y$ ) remains unaltered at filling transition, since the free energy variation due to the increase in the gas-liquid interfacial length, $2 \sigma_{l v} \Delta \ell(y) / \tan \alpha$, is exactly balanced by the decrease of the surface free energy associated with the liquid-substrate interface, $2\left(\sigma_{l w}-\sigma_{v w}\right) \Delta \ell(y) / \sin \alpha=-2 \sigma_{l v} \cos \theta / \sin \alpha$, where $\sigma_{l v}, \sigma_{l w}$ and $\sigma_{v w}$ are the surface tensions associated with the vapour-liquid, liquid-substrate and 
vapour-substrate interfaces, respectively. This fact originates the emergence of breather-mode soft modes near the filling transition $[5,6]$. Consequently, next-to-leading contributions to the excess surface free energy, such as binding potential contributions, will control the interfacial behaviour. For short-ranged forces, where the binding potential is irrelevant, the interfacial phenomenology is determined by the line free energies associated with the three-phase liquid-vapour-substrate lines and to the wedge bottom when the interface is either pinned at the wedge or away from it [15]. These line free energies are associated with deviations of the density profiles with respect to the flat surface ones, induced by three-phase coexistence and enhanced packing effects or stronger interactions near the wedges. At a mean-field level, we expect that, if the line free energy associated with a wedge in contact with the vapour is smaller than the sum of the three-phase line free energies and the line free energy associated with a wedge in contact with the liquid, then the gas-liquid interface will be pinned at the wedge bottom. Otherwise, the interface will unbind from the wedge. In this sense, the difference of total line free energies for the different considered situations can be understood as a pinning contact potential for the gas-liquid interface. Interfacial fluctuations shift the unbinding value for the pinning potential strength [13-15]. In fact, the observed phenomenology in the wedge filling transition for short-ranged forces at bulk coexistence is analogous to $2 \mathrm{D}$ random-bond critical wetting, where the pinning contact potential plays the role of the temperature, and $\theta-\alpha$ acts as an ordering field [13]. The borderline between first-order and critical filling corresponds to a tricritical point, with the strength of the fluid-solid interactions close to the wedge axis as a new relevant operator. However, along the constant strength path the tricritical exponents eq. (1) are the same as in critical filling [15], so the observation of tricritical filling may be elusive.

Critical filling transitions have been observed unambiguously in computer simulation studies of the 3D Ising model [21-24]. The double-wedge geometry with applied antisymmetric surface fields is specially suitable to make a systematic finite-size characterization of the critical behavior of the filling transition, confirming the critical exponents (1) obtained from interfacial Hamiltonian models $[5,6]$. In this letter we consider a suitable modification of this system in our search for the tricritical filling transition, where a line field opposite to the surface field is applied near each wedge (see fig. 1). In this way different magnetizations are favoured on nearest-neighbour sites close to the wedges, which eventually may induce the pinning of the two-phase interface at the wedges. We expect that the tricritical filling transition may be observed by tuning the line field. For this purpose, we perform Monte Carlo simulations for different box sizes, and the tricritical filling transition is located by the matching of the magnetization probability distribution functions (PDFs) with the theoretical prediction of the phenomenological model [24].

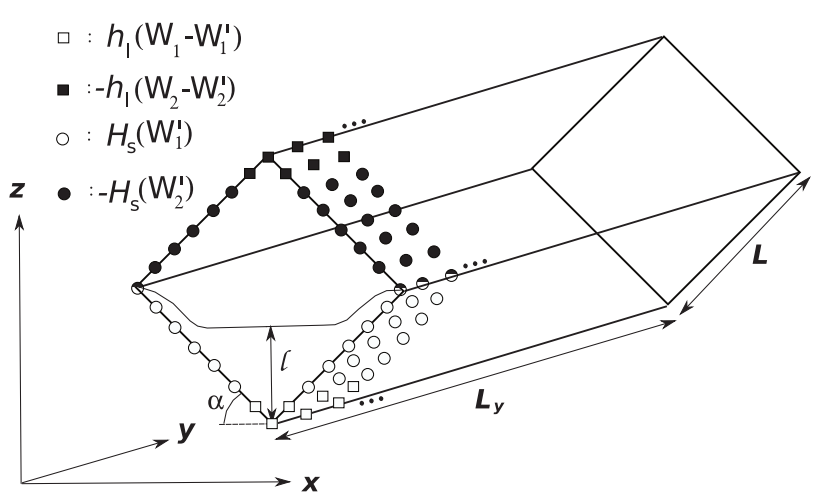

Fig. 1: Schematic picture of the modified antisymmetric double-wedge geometry of size $L \times L \times L_{y}$ and characterized by a tilt angle $\alpha$. Filled and empty symbols refer to the spins associated with the surfaces which define the $W_{1}$ (filled) and $W_{2}$ (empty) wedges. Circles represent the sites subject to the surface fields $H_{s}\left(W_{1}^{\prime}\right)$ and $-H_{s}\left(W_{2}^{\prime}\right)$. Squares represent the wedge sites where act the line field $h_{l}\left(W_{1}-W_{1}^{\prime}\right)$ and $-h_{l}\left(W_{2}-W_{2}^{\prime}\right)$.

First we will obtain the analytical form of the tricritical magnetization PDF for the antisymmetric double-wedge geometry within the breather-mode model. This model assumes that critical (and tricritical) filling phenomena at bulk coexistence can be understood using an effective pseudo-one-dimensional wedge Hamiltonian which accounts only for breather-mode excitations $[5,6]$ :

$$
\mathcal{H}_{W}[\ell]=\int \mathrm{d} y\left\{\frac{\Lambda(\ell)}{2}\left(\frac{\mathrm{d} \ell}{\mathrm{d} y}\right)^{2}+V_{W}(\ell)\right\},
$$

where $\ell(y)$ is the local height of the interface above the wedge bottom. For the double-wedge geometry characterized by a tilt angle $\alpha, 0<\ell(y)<2 L \sin \alpha$. The effective bending term $\Lambda(\ell)$ resisting fluctuations along the wedge can be expressed as

$$
\Lambda(\ell)=\frac{2 \Sigma}{\tan \alpha} \min (\ell, 2 L \sin \alpha-\ell) .
$$

For systems with short-ranged forces, the effective binding potential $V_{W}(\ell)$ has a short-ranged contribution, which can be modeled as a contact potential of strength $-U$ at $\ell=0$ and $\ell=2 L \sin \alpha$, and a long-range part which arises from the surface energy cost of creating an interfacial configuration:

$$
V_{W}(\ell)=\Lambda(\ell)\left(1-\frac{\cos \theta}{\cos \alpha}\right) .
$$

By setting $k_{B} T=1$ for convenience, the partition function $Z\left(\ell_{b}, \ell_{a}, L_{y}\right)$ can be represented as a path integral of $\exp \left(-\mathcal{H}_{W}\right)$ over all the paths which start for $y=0$ at an interfacial height $\ell_{a}$ and end at interfacial height $\ell_{b}$ for $y=L_{y}[30]$. Due to the presence of a position-dependent stiffness coefficient, some care is required in the definition of the partition function and its measure [8], similar to the factor-ordering problem in solid-state quantum 
mechanics when there is an effective position-dependent mass $[31,32]$. We follow the prescription proposed in earlier work which provides a solution which is mathematically consistent and agrees with necessary thermodynamic requirements $[13-15]$. The partition function $Z\left(\ell_{b}, \ell_{a}, L_{y}\right)$ can be expressed as [30]

$$
Z\left(\ell_{b}, \ell_{a}, L_{y}\right)=\sum_{i} \psi_{i}\left(\ell_{b}\right) \psi_{i}^{*}\left(\ell_{a}\right) \mathrm{e}^{-E_{i} L_{y}},
$$

where $\left\{\psi_{i}(\ell)\right\}$ is a complete orthonormal set of eigenfunctions with associated eigenvalues $E_{i}$ of the Hamiltonian operator $H_{W}$,

$$
H_{W} \equiv-\frac{1}{2} \frac{\partial}{\partial \ell_{b}}\left[\frac{1}{\Lambda\left(\ell_{b}\right)} \frac{\partial}{\partial \ell_{b}}\right]+V_{W}\left(\ell_{b}\right)+\tilde{V}_{W}\left(\ell_{b}\right) .
$$

Here the term $\tilde{V}_{W}(\ell)$ is given by

$$
\tilde{V}_{W}(\ell)=-\frac{1}{2 \Lambda(\ell)}\left[\frac{3}{4}\left(\frac{\Lambda^{\prime}(\ell)}{\Lambda(\ell)}\right)^{2}-\frac{\Lambda^{\prime \prime}(\ell)}{2 \Lambda(\ell)}\right],
$$

where the prime denotes the derivative with respect to its argument. The short-ranged contribution to $V_{W}(\ell)$ leads to boundary conditions for the eigenvalue problem. So, at $\ell=0$, we force the eigenfunctions to have the same short-distance expansion as in the infinite wedge situation $[15,24]$

$$
\psi(\ell) \sim \sqrt{\ell} \pm \frac{\Gamma[-1 / 3] 3^{-2 / 3}}{\Gamma[1 / 3]} \frac{\ell^{3 / 2}}{\xi_{u}},
$$

where $\xi_{u} \sim\left|U-U_{t c}\right|^{-1}$ is a characteristic length associated with the contact potential at the wedge bottom and the positive and negative signs corresponds to $U>U_{t c}$ (firstorder filling) and $U<U_{t c}$ (critical filling), respectively, $U_{t c}$ being the tricritical value [15]. An analogous boundary condition is applied at the upper boundary $\ell=2 L \sin \alpha$, substituting $\ell$ by $2 L \sin \alpha-\ell$ in eq. (8).

For the case of periodic boundary conditions the interfacial partition function in the double wedge $Z_{p}$ can be obtained as

$$
Z_{p}\left(L, L_{y}\right)=\int_{0}^{2 L \sin \alpha} Z\left(\ell, \ell, L_{y}\right) \mathrm{d} \ell=\sum_{i} e^{-E_{i} L_{y}}
$$

and the PDF for the interfacial height as [24]

$$
P_{W}\left(\ell, L, L_{y}\right)=\frac{\sum_{i}\left|\psi_{i}(\ell)\right|^{2} e^{-E_{i} L_{y}}}{\sum_{i} e^{-E_{i} L_{y}}} .
$$

At the tricritical point (i.e. $\left.\theta=\alpha, \xi_{u} \rightarrow \infty\right)$, the eigenfunctions are alternating even and odd functions with respect to $\ell=L \sin \alpha$, which have in the interval $[0, L \sin \alpha]$ the expression

$$
\psi_{i}(\ell) \propto \sqrt{\ell}\left[\mathrm{Ai}\left(\left(-4 \epsilon_{i}\right)^{1 / 3} \ell\right)+\frac{1}{\sqrt{3}} \mathrm{Bi}\left(\left(-4 \epsilon_{i}\right)^{1 / 3} \ell\right)\right],
$$

Table 1: First solutions of eq. (12).

\begin{tabular}{ccc}
\hline$i$ & $x_{i}$ & Eigenfunction parity \\
\hline 0 & 0.0000 & Even \\
1 & -1.9864 & Odd \\
2 & -2.9488 & Even \\
3 & -3.8253 & Odd \\
4 & -4.5781 & Even \\
5 & -5.2956 & Odd \\
6 & -5.9503 & Even \\
7 & -6.5843 & Odd \\
8 & -7.1779 & Even \\
\hline
\end{tabular}

where $\operatorname{Ai}(x)$ and $\operatorname{Bi}(x)$ are Airy functions, and the reduced eigenvalue $\epsilon_{i}=\Sigma E_{i} / \tan \alpha$. Note that the eigenfunctions $\psi_{i}(\ell)$ satisfy the boundary conditions eq. (8) for $\ell=0$ and the analogous boundary condition at $\ell=2 L \sin \alpha$. On the other hand, the even eigenfunctions show a kink at $\ell=$ $L \sin \alpha$, consequence of the Dirac-delta term $\Lambda^{\prime \prime}(x)$ in $\tilde{V}_{W}$ (see eqs. (3) and (7)), while the odd eigenfunctions vanish at $\ell=L \sin \alpha$. Thus the eigenvalues can be obtained by imposing the vanishing at $\ell=L \sin \alpha$ of the eigenfunction (odd case) or the derivative of $\psi(\ell) / \sqrt{\ell}$ (even case, see eq. (21) in ref. [24]). So, the associated eigenvalues have the expression $E_{i}=-\tan \alpha x_{i}^{3} / 4 \Sigma(L \sin \alpha)^{3}$, where $x_{i}$ are the solutions of the transcendental equations:

$$
-\frac{1}{\sqrt{3}}= \begin{cases}\frac{\operatorname{Ai}^{\prime}(x)}{\operatorname{Bi}^{\prime}(x)}, & \psi(\ell) \text { even }, \\ \frac{\operatorname{Ai}(x)}{\operatorname{Bi}(x)}, & \psi(\ell) \text { odd },\end{cases}
$$

which can be solved numerically or graphically. Table 1 shows the first few solutions to eq. (12).

In order to compare these predictions with the existing Ising model computer simulation results, we must convert the dependence on the interfacial height into an appropriate microscopic observable. For Ising model this is the local magnetization density $m$ in the vertical plane at position $y$ along the wedge, which in the breather-mode picture can be related to $\ell$ as $[24,33]$ :

$$
\frac{m}{m_{b}}= \begin{cases}\left(\frac{\ell}{L \sin \alpha}\right)^{2}-1, & 0<\ell<L \sin \alpha \\ 1-\left(\frac{2 L \sin \alpha-\ell}{L \sin \alpha}\right)^{2}, & L \sin \alpha<\ell<2 L \sin \alpha\end{cases}
$$

where $m_{b}>0$ is the bulk magnetization density. The magnetization PDF is then related to the interfacial height PDF via $P_{W}(m)=P_{W}(\ell(m))|\mathrm{d} \ell / \mathrm{d} m|$. At the tricritical filling transition, the magnetization $\mathrm{PDF}$ is given by

$$
\text { see eq. (14) on the next page }
$$

where $N_{i}$ are the eigenfunction normalization factors and $\kappa=\tan \alpha /\left(4 \Sigma \sin ^{3} \alpha\right)$. Note that the PDF exactly at tricritical filling, as well as at critical filling [24], does not 


$$
P_{W}(m)=\frac{\sum_{i=0}^{\infty} N_{i}^{2}\left[\mathrm{Ai}\left(-x_{i} \sqrt{1-\frac{|m|}{m_{b}}}\right)+\frac{1}{\sqrt{3}} \operatorname{Bi}\left(-x_{i} \sqrt{1-\frac{|m|}{m_{b}}}\right)\right]^{2} e^{\kappa x_{i}^{3} \frac{L_{y}}{L^{3}}}}{\sum_{i=0}^{\infty} e^{\kappa x_{i}^{3} \frac{L_{y}}{L^{3}}}},
$$

depend on $L$ and $L_{y}$ independently, but via the scaling combination $L_{y} / L^{3}$, i.e. $P_{W}\left(m, L, L_{y}\right)=P_{W}\left(m, L_{y} / L^{3}\right)$, in agreement with previous scaling arguments [21-23]. For $L^{3} / L_{y}=0$, the magnetization PDF over the interval $\left[-m_{b}, m_{b}\right]$ is flat, and as $L^{3} / L_{y}$ increases, the magnetization PDF becomes bimodal, where the most probable magnetization density in each section corresponds to $m= \pm m_{b}$.

In order to confirm the predicted existence of a tricritical filling transition, we performed Monte Carlo simulations for a nearest-neighbor Ising model (isomorphic to a lattice gas) on a simple cubic lattice with linear dimensions $L \times L \times L_{y}$ in lattice spacing units. Periodic boundary conditions are applied along the $y$-direction, and in the remaining boundaries free boundary conditions are applied. We set similar bulk conditions as those previously considered for the critical filling characterization [21-24]. So, the Hamiltonian of the Ising model is given by

$$
\begin{aligned}
\beta H= & -\frac{1}{4} \sum_{\langle i, j\rangle_{\text {bulk }}} S_{i} S_{j}-\frac{1}{8} \sum_{\langle i, j\rangle \in W_{1} \cup W_{2}} S_{i} S_{j} \\
& -\beta H_{s} \sum_{i \in W_{1}^{\prime}} S_{i}+\beta H_{s} \sum_{i \in W_{2}^{\prime}} S_{i} \\
& -\beta h_{l} \sum_{i \in W_{1}-W_{1}^{\prime}} S_{i}+\beta h_{l} \sum_{i \in W_{2}-W_{2}^{\prime}} S_{i} .
\end{aligned}
$$

As in earlier works, we choose $\beta J \equiv J / k_{B} T=1 / 4$ and the surface exchange constant $J_{s}=J / 2$. Under these conditions, the simulation box is a double wedge characterized by a tilt angle $\alpha=\pi / 4$, the bulk magnetization is $m_{b} \approx 0.75$, and $\Sigma=\beta \sigma a^{2} \approx 0.0981$, with $\sigma$ being the interfacial tension of the Ising model and $a$ the lattice spacing [22]. We define $W_{1}$ and $W_{2}$ as the two neighbouring $L \times L_{y}$ free surfaces which meet at each wedge (see fig. 1). Focussing on $W_{1}$, the surface field $H_{s}$ is applied in a set of sites $W_{1}^{\prime}$ of $W_{1}$ which are away from the wedge, and a line field $h_{l}$ on the remaining sites. For the antisymmetric setup, opposite fields $-H_{s}$ and $-h_{l}$ are applied on $W_{2}^{\prime}$ and $W_{2}-W_{2}^{\prime}$, respectively, where the set $W_{2}^{\prime}$ is the mirror image of $W_{1}^{\prime}$ with respect to the diagonal symmetry plane of the simulation box. We have some freedom to define the set of sites where the line field $h_{l}$ is applied. We impose as a condition that the sites must be at or very close to the wedge, so $H_{s}$ is applied on most of the sites of the free surfaces. Thus the stripped regions of microscopic width where $h_{l}$ acts reduce to the wedge axes for large $L$. The value of $H_{s}$ determines the contact angle, and for a flat substrate the wetting transition is critical [34]. On the other hand, the introduction of $h_{l}$ alters the value of the pinning potential in such a way that, if $h_{l}$ and $H_{s}$ are of opposite sign, opposite magnetizations on sites close to the wedge are favoured, and this fact may eventually induce the interfacial pinning at the wedges. So, $h_{l}$ will contribute to the pinning contact potential, but we cannot simply identify it with the pinning potential strength, as the other coupling parameters contribute to it. The connection with the phenomenological model is now apparent. However, we do not observe tricriticality for every choice of $W_{1}^{\prime}$ and $W_{2}^{\prime}$. For example, if $h_{l}$ is applied solely on the sites along the wedge, only critical filling is observed [35]. Analogously, if we try to favour the interfacial pinning by weakening the exchange coupling of the wedge sites on the surface, non-ergodic behaviour is observed before the filling transition may become tricritical [35]. In the present work, we consider that the line field $h_{l}$ acts on the sites which are along the wedge and their nearest neighbours (see fig. 1). These Monte Carlo simulations were performed by using the standard Metropolis algorithm [36]. The quantities we are interested in equilibrate quite slowly, so we considered runs of order of $10^{8}$ sweeps, where a sweep is $L^{2} \times L_{y}$ attempted updates of a spin chosen at random. In each simulation we evaluated the magnetization PDF, where $m$ is defined as the average over the spins at each slice which are neither nearest nor next-nearest neighbors to $W_{1}$ or $W_{2}$ in order to minimize the effect of the enhanced order close to the surfaces. The values of $H_{s}$ are taken to be the apparent critical filling values for each box size and $h_{l}=H_{s}$ [24] (in all cases $H_{s} \approx 0.72$ ). Note that the filling transition always occurs for $\theta=\alpha$ and the value of $H_{s}$ determines the value of the contact angle $\theta$, regardless of the value of $h_{l}$. However, we have used single-histogram reweighting techniques $[37,38]$ to tune the dependence on $H_{s}$ close to the filling transition of the magnetization PDF at a given value of $h_{l}$. In order to characterize finite-size effects, we perform the simulations for different system sizes, in such a way that the ratio $L_{y} /(L-4)^{3}$ is approximately constant: $19 \times 19 \times 16,24 \times 24 \times 37,34 \times 34 \times 124$ and $44 \times 44 \times 294$ (the ratio $L_{y} /(L-4)^{3}$ is approximately equal to 0.0046 ). Recall that the predicted critical and tricritical magnetization PDFs depend only on this size ratio.

Figure 2 shows typical magnetization PDFs for different values of $H_{s}$ and $h_{l}$. For all values of $h_{l}$, the PDF is bimodal for values of $H_{s}$ well below the filling transition value, with maxima localized approximately at $\pm m_{b}$. On the other hand, if $H_{s}$ is well above the filling transition value, the PDF becomes unimodal with a single maximum at $m=0$. Differences are observed when $H_{s}$ is around the filling transition value. For $h_{l}=H_{s}$, we reproduce the results already presented elsewhere [24], confirming that, 

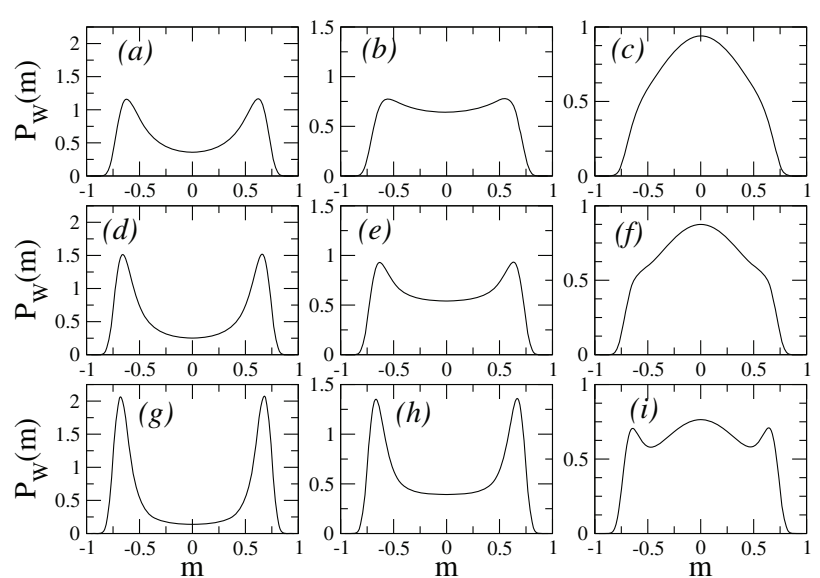

Fig. 2: Magnetization PDFs for $L=24, L_{y}=37$ and different values of $\left(H_{s}, h_{l}\right)$ : (a) $(0.7084,0.7284)$, (b) $(0.7284,0.7284)$, (c) $\quad(0.7484,0.7284), \quad(d) \quad(0.7084,0), \quad$ (e) $\quad(0.7284,0)$, (f) $(0.7484,0), \quad$ (g) $(0.7084,-0.5)$, (h) $(0.7284,-0.5)$, and (i) $(0.7484,-0.5)$.

under these conditions, the filling transition is critical. Moreover, at the critical filling value of $H_{s}$ there is an excellent match with the predicted critical filling magnetization PDF from the phenomenological theory. If we set $h_{l}=-0.5$, a different scenario is observed. The magnetization PDFs for different box sizes do not match the theoretical critical filling magnetization PDF. Actually, the location of the maxima is quite insensitive to the value of $H_{s}$, and for large $H_{s}$ we observe a trimodal PDF with an additional maximum at $m=0$. By increasing $H_{s}$, the relative PDF height of the maxima at $m \approx \pm m_{b}$ with respect to the PDF value at $m=0$ decreases, until the former disappear. These observations are an indication that the filling transition may be of first order for $h_{l}=-0.5$. The coexisting phases would be a pinned interfacial state, characterized by the bimodal PDF similar to the observed one for small $H_{s}$, and the unbound interfacial state, characterized by the unimodal PDF observed for large $H_{s}$. Near the first-order transition, the magnetization PDF will be a linear superposition between the coexisting phases PDFs, where their relative weights are related to the deviation of $H_{s}$ with respect to the transition value. However, our simulations show the rounding of this transition for the considered system sizes, since the PDFs of the coexisting phases overlap considerably. We explored the values of $h_{l}$ between $h_{l}=-0.5$ and $h_{l}=0.72$ to locate the borderline between these two scenarios, that we expect to be a tricritical point from our theoretical analysis. The procedure to locate the tricritical point is as follows. As the simulation PDFs show tails for large $|m|$ (due to capillary fluctuations or other irrelevant fluctuations), we match unnormalized PDFs (i.e. multiplied by an unknown factor to be determined in the matching procedure) to the theoretical expression eq. (14) in a magnetization window $|m|<m_{\text {cut }}$. For our simulations, we choose $m_{\text {cut }}=0.5$, finding the tricritical filling transition at $h_{l} \approx 0$. Figure 3 shows the best

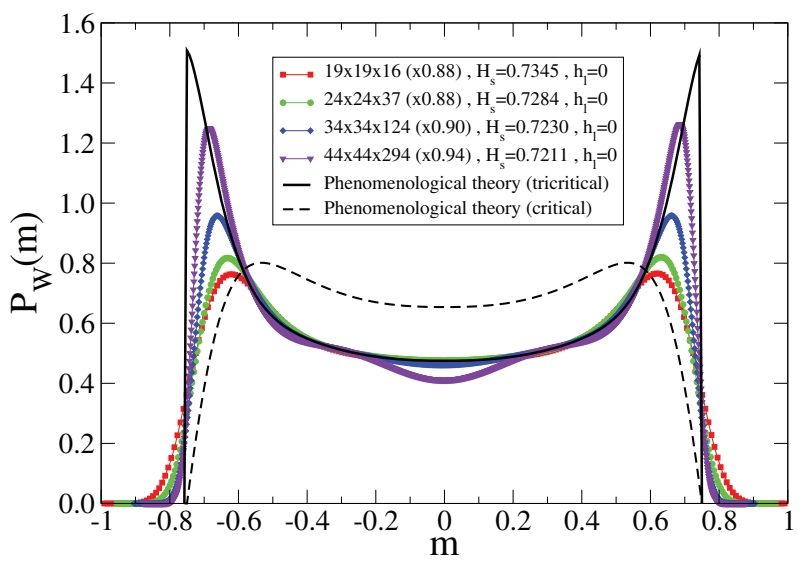

Fig. 3: (Color online) Plot of the magnetization PDFs for $h_{l}=$ 0 and: $H_{s}=0.7345,19 \times 19 \times 16$ (squares); $H_{s}=0.7284$, $24 \times 24 \times 37$ (circles); $H_{s}=0.7230,34 \times 34 \times 124$ (diamonds); and $H_{s}=0.7211,44 \times 44 \times 294$ (triangles). The continuous line corresponds to the breather-mode model predicted tricritical filling PDF, and the dashed line to the predicted critical filling PDF [24].

matching magnetization PDFs for different simulation box sizes and $h_{l}=0$. The values of $H_{s}$ correspond approximately to the transition values for critical filling, indicating that the filling transition boundary is unaffected by the field $h_{l}$. On the other hand, the PDFs are clearly different from the critical filling PDF, and as $L$ increases the two maxima converge to the theoretical tricritical PDF. This is the main result of our paper, being a clear indication of the existence of a tricritical filling transition. Finally, it is worth noting that the magnetization PDF for the largest system seems to deviate from the theoretical prediction for small values of $|m|$. This is also observed for critical filling [35]. We explain these discrepancies by the breakdown of the breather-mode picture for small values of $|m|$ and large $L$. In fact, the analysis of typical snapshots shows tilted configurations when $\ell \approx L / \sqrt{2}$, indicating that tilt and torsional modes [7] may be important under these conditions.

In conclusion, we have found strong evidences that the filling transition can be tricritical by introducing a local field along the wedges which localizes the interface. To demonstrate this, we have performed Monte Carlo simulations of the 3D Ising model in a double-wedge geometry with applied antisymmetric surface fields and an additional field acting along the wedges. A finite-size analysis of these simulations is in agreement with the predictions of the breather-mode model for the tricritical filling. Although our study is restricted to the case of short-ranged forces, it may be relevant for the case of dispersive forces, since the breather-mode model predicts that the filling transition may also become of first order, with a critical end point as the borderline with the critical filling regime [15]. We expect that the predictions for the latter case may be confirmed experimentally. 


$$
* * *
$$

We thank Prof. K. Binder, Prof. A. O. Parry, Dr. E. V. Albano and Dr. C. RAscón for discussions and reading of the manuscript. AR-R thanks the Institut für Physik (Johannes Gutenberg-Universität Mainz) for hospitality during a research stay where this work was begun. AR-R, JMR-E and LFR acknowledge financial support from the Spanish Ministerio de Economía y Competitividad through grants No. FIS2009-09326 and FIS2012-32455, and Junta de Andalucía through grant No. P09-FQM-4938, all co-funded by the EU FEDER, and the Portuguese Foundation for Science and Technology under Contract No. EXCL/FIS-NAN/0083/2012.

\section{REFERENCES}

[1] Gau H., Herminghaus S., Lenz P. and Lipowsky R., Science, 283 (1999) 46.

[2] Rascón C. and Parry A. O., Nature, 407 (2000) 986.

[3] Bruschi L., Carlin A. and Mistura G., Phys. Rev. Lett., 89 (2002) 166101.

[4] Rejmer K., Dietrich S. and Napiórkowski M., Phys. Rev. E, 60 (1999) 4027.

[5] Parry A. O., Rascón C. and Wood A. J., Phys. Rev. Lett., 85 (2000) 345.

[6] Parry A. O., Wood A. J. and Rascón C., J. Phys.: Condens. Matter, 13 (2001) 4591.

[7] Greenall M. J., Parry A. O. and Romero-Enrique J. M., J. Phys.: Condens. Matter, 16 (2004) 2515.

[8] Bednorz A. and Napiónkowski M., J. Phys. A: Math. Gen., 33 (2000) L353.

[9] Henderson J. R., J. Chem. Phys., 120 (2004) 1535.

[10] Henderson J. R., Phys. Rev. E, 69 (2004) 061613.

[11] Henderson J. R., Mol. Simul., 31 (2005) 435.

[12] Rascón C. and Parry A. O., Phys. Rev. Lett., 94 (2005) 096103.

[13] Romero-Enrique J. M. and Parry A. O., Europhys. Lett., 72 (2005) 1004.

[14] Romero-Enrique J. M. and Parry A. O., J. Phys.: Condens. Matter, 17 (2005) S3487.

[15] Romero-Enrique J. M. and Parry A. O., New J. Phys., 9 (2007) 167.
[16] Parry A. O. and Rascón C., J. Phys.: Condens. Matter, 23 (2011) 015004.

[17] Bernardino N. R., PArry A. O. and RomeroEnrique J. M., J. Phys.: Condens. Matter, 24 (2012) 182202.

[18] Bruschi L., Carlin A. and Mistura G., J. Chem. Phys., 115 (2001) 6200.

[19] Bruschi L., Carlin E. and Mistura G., J. Phys.: Condens. Matter, 15 (2003) S315.

[20] Bruschi L., Carlin A., Parry A. O. and Mistura G., Phys. Rev. E, 68 (2003) 021606.

[21] Milchev A., Muller M., Binder K. and Landau D. P., Phys. Rev. Lett., 90 (2003) 136101.

[22] Milchev A., Muller M., Binder K. and Landau D. P., Phys. Rev. E, 68 (2003) 031601.

[23] Binder K., Muller M., Milchev A. and Landau D. P., Comput. Phys. Commun., 169 (2005) 226.

[24] Romero-Enrique J. M., Rodríguez-Rivas A., Rull L. F. and Parry A. O., Soft Matter, 9 (2013) 7069.

[25] MaliJevsky A. and Parry A. O., Phys. Rev. Lett., 110 (2013) 166101.

[26] Malijevsky A. and Parry A. O., J. Phys.: Condens. Matter, 25 (2013) 305005.

[27] Concus P. and Finn R., Proc. Natl. Acad. Sci. U.S.A., 63 (1969) 292.

[28] Pomeau Y., J. Colloid Interface Sci., 113 (1986) 5.

[29] Hauge E. H., Phys. Rev. A, 46 (1992) 4994

[30] Burkhardt T. W., Phys. Rev. B, 40 (1989) 6987.

[31] Thomsen J., Einevoll G. T. and Hemmer P. C., Phys. Rev. B, 39 (1989) 12783.

[32] Chetouani L., Dekar L. and Hammann T. F., Phys. Rev. A, 52 (1995) 82.

[33] Albano E. V., Virgilits A. D., Müller M. and Binder K., J. Phys.: Condens. Matter, 15 (2003) 333.

[34] Binder K., Landau D. P. and Wansleben S., Phys. Rev. B, 40 (1989) 6971.

[35] Rodríguez-Rivas A., Romero-Enrique J. and Rull L. F., unpublished (2014).

[36] Metropolis N., Rosenbluth A. W., Rosenbluth M. N., Teller A. N. and Teller E., J. Chem. Phys., 21 (1953) 1087.

[37] Ferrenberg A. M. and Swendsen R. H., Phys. Rev. Lett., 61 (1988) 2635.

[38] Ferrenberg A. M. and Swendsen R. H., Phys. Rev. Lett., 63 (1989) 1195. 